\title{
Visceral pseudoaneurysms and the role of endovascular treatment - a case report
}

\begin{abstract}
Introduction: Visceral pseudoaneurysms are pathological dilations of the visceral arteries and/or their branches. They are a rare entity but with devastating consequences given their high potential for rupture and hemorrhage. The evolution of endovascular techniques has changed the paradigm in the treatment of this entity, making it the preferred option for the elective treatment of visceral pseudoaneurysms.
\end{abstract}

Clinical case: The authors described the case of a pancreatic pseudoaneurysm in a young male patient, with past medical history of chronic pancreatitis and pancreatic pseudocyst, marked smoking and alcoholic habits, which presented with an abdominal pain and a drop in hemoglobin. After discussing the case with the Vascular Surgery department, it was decided towards an endovascular treatment given the patient's clinical stability and appropriate anatomical location for the proposed intervention. Coil embolization via humeral artery was performed with immediate angiographic success, and clinical, analytical and imaging improvement in the postoperative period.

Conclusion: In addition to open surgical repair and laparoscopic surgery, there are also endovascular procedures for the treatment of visceral pseudoaneurysms, so the vascular surgeon must be aware with the available strategies, taking into account the patient, the characteristics and location of the visceral pseudoaneurysm.

Keywords: visceral pseudoaneurysm, pancreatic pseudoaneurysm, embolization

\author{
Volume 5 Issue 6 - 202I
}

\author{
Adriana Figueiredo, Nelson Camacho, Maria \\ Emília Ferreira \\ Serviço de Cirurgia Vascular, Hospital de Santa Marta - Centro \\ Hospitalar Universitário Lisboa Central, Portugal \\ Correspondence: Nelson Camacho, Serviço de Cirurgia \\ Vascular, Hospital de Santa Marta - Centro Hospitalar \\ Universitário Lisboa Central, Portugal, \\ Email nelsongcamacho@gmail.com
}

Received: October 15, 202 I | Published: November 16, 202

\section{Introduction}

Visceral (or splanchnic) pseudoaneurysms are pathological dilations that affect the visceral arteries or their branches including the arteries that derive from the celiac trunk, the superior and inferior mesenteric artery. ${ }^{1}$ Unlike a true aneurysm, which is a focal dilatation that involves all layers of the artery, pseudoaneurysms (also known as false aneurysms) represent a disruption in the arterial wall continuity with patent flow in a defined space beyond the arterial wall. Both have an increased risk of rupture but due to the lower structural stability of pseudoaneurysms, they are more likely to rupture than true aneurysms. ${ }^{2}$

As a whole visceral pseudoaneurysms (VPA) are a rare entity but with devastating consequences if left untreated, given their high potential for rupture and potentially fatal hemorrhage. ${ }^{1}$ VPA typically result from iatrogenic injury during surgery or endovascular procedures, vasculitis, or states of inflammation or infection, as in pancreatitis. ${ }^{3,4}$ Splenic artery pseudoaneurysms are the most commonly found in association with pancreatic disease given their proximity to the pancreas, with chronic pancreatitis with secondary pseudocyst formation being the main cause of pancreatic pseudoaneurysms. ${ }^{5,6}$ Due to the action of digestive enzymes on the artery, the pseudocyst is incorporated as a part of the arterial wall and its rupture converts the pseudocyst into a pseudoaneurysm. However, pancreatic pseudoaneurysms can also occur in the absence of a pseudocyst. ${ }^{7}$

The clinical presentation depends on the location with no specific signs or symptoms although most VPA are asymptomatic or incidental findings. ${ }^{6,8} \mathrm{Abdominal}$ pain is the most common symptom of unruptured pseudoaneurysms followed by jaundice, nausea and vomiting, or delayed gastric emptying. When rupture occurs it can present as hematemesis, melena, hematochezia, haemobilia or retroperitoneal hemorrhage depending on the location of the lesion. Pain occurs in only one third of ruptures, with hypotension and hemorrhagic shock frequently being the only clinical manifestation. ${ }^{1,2,4}$

Due to its rarity, the hypothesis of visceral pseudoaneurysm is not included at the initial investigation of a patient with nonspecific abdominal complaints causing a delay in its diagnosis and treatment.

VPA are currently most often diagnosed because of the increased access and use of different forms of intra-abdominal imaging which allows the identification of occult and asymptomatic lesions as well as the planning and decision of the most suitable treatment for each situation, taking into account the particular anatomy of the false aneurism, their location, any associated clinical conditions and the underlying condition of the patient. ${ }^{3}$

In addition to the high potential for rupture, pseudoaneurysms tend to grow rapidly after the initial insult. ${ }^{4}$ Because of the unstable nature of this entity, VPA should be promptly treated upon diagnosis regardless of size., ${ }^{3,10}$ The progress of the endovascular techniques has determined a paradigm shift in the treatment of this entity, with early elective treatment through endovascular techniques being the preferred option for the treatment of visceral pseudoaneurysms rather than open or laparoscopic surgery. $2,3,9,10$ Improvements in endovascular therapies have also allowed an enhanced ability to treat anatomically complex and difficult to reach pseudoaneurysms by a large variety of individualized and precise catheter-based therapies. Endovascular techniques are associated with high success rate and lower morbidity and mortality than conventional surgery and include coil embolization of the native artery or the pseudoaneurysm, covered stents or multilayer stents. , $3,8,11,12^{2}$

In this paper we present a case of a pancreatic pseudoaneurysm - a false aneurysm that affects the pancreatic and peripancreatic arteries - which is mainly caused by recurrent pancreatitis. They typically 
affect younger patients, mostly men with a history of heavy alcohol consumption and a history of recurrent chronic pancreatitis with associated pancreatic pseudocyst.

\section{Clinical case}

We present the case of a 48-year-old male with a known personal history of chronic recurrent pancreatitis and pancreatic pseudocyst, chronic gastritis, multifactorial anemia with stable values $(\mathrm{Hb} 7.6-7.9$ $\mathrm{g} / \mathrm{dL}$ ) and significant alcohol and smoking habits. He was admitted to the emergency department due to epigastric abdominal pain, nausea, anorexia and fever. After investigation, he was hospitalized due to acalculous acute chronic pancreatitis (analytically without severity criteria). On the fourth day of hospitalization, due to worsening of the abdominal pain and a drop in hemoglobin $(\mathrm{Hb} 7.5 \mathrm{~g} / \mathrm{dl}->6.6 \mathrm{~g} / \mathrm{dl})$, he performs abdominal computed tomography angiography (CTA) Figure $1 \& 2$.
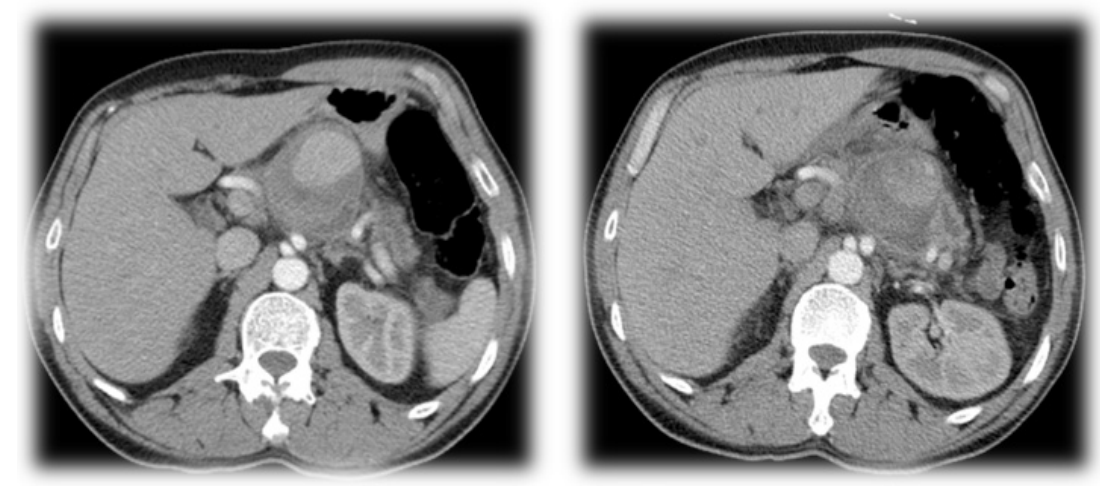

Figure I \& 2 Pre-operative CTA showing a 'rounded structure with enhanced vascular pattern along the small gastric curvature, measuring around I5 mm in diameter. Around this structure coexists a hematic collection that extends to the epiploic retrocavity measuring $6.1 \times 4.4 \mathrm{~cm}$. No evidence of active bleeding.

Because of the patient's clinical stability and the imaging suspicion of pseudoaneurysm in dependence on the left gastric artery, endovascular treatment was decided after discussion with the Vascular Surgery department.

It was performed coil embolization of the branch responsible for the false aneurysm by left humeral artery and under general anesthesia with satisfactory final angiographic result and no anesthetic-surgical complications. The patient was favorable postoperative evolution with clinical and analytical improvement: relief of the abdominal complaints and stable hemoglobin values Figure $3,4 \& 5$. Control CTA was performed at $72 \mathrm{~h}$ postoperative, confirming the success of the procedure (Figure 6).
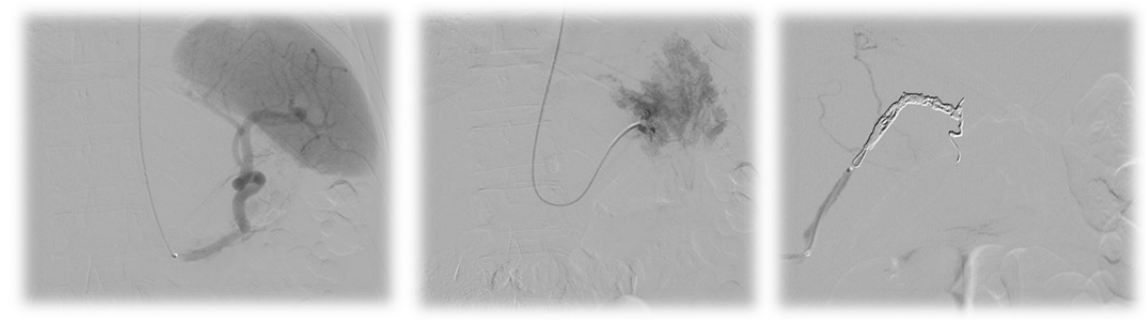

Figure 3,4 \& 5 Steps from the vascular intervention. 3- Splenic artery catheterization without evidence of contrast extravasation. 4- Catheterization of the left gastric artery and evidence of an exuberant flush of this vessel. 5 - Embolization of the artery with Concertoß $5 \mathrm{~mm}$ and $2 \times 10 \mathrm{~mm}$ detachable coils.

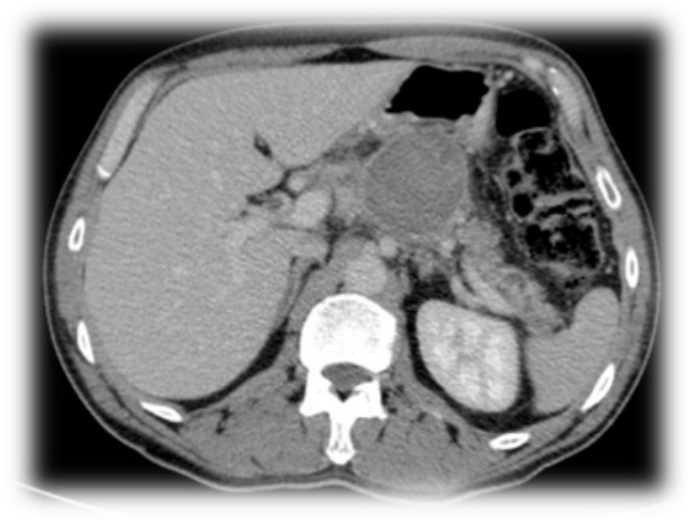

Figure 6 Control CTA after the procedure showing embolization coil in the topography of the left gastric artery and no contrast extravasation.

\section{Discussion}

Vascular complications associated with chronic pancreatitis are one of the main causes of morbidity and mortality and mainly caused by the erosion and rupture of a pseudoaneurysm. ${ }^{7}$ With greater availability and use of imaging diagnostic modalities, occult visceral pseudoaneurysms are increasingly diagnosed which allows their early elective treatment and thus avoiding their devastating complications. Given their rarity and because VPA are mostly asymptomatic or associated with unspecific abdominal symptoms, the diagnostic hypothesis of visceral pseudoaneurysm does not enter into the initial investigation of patients with abdominal complaints or malaise. However, in the presence of a patient with a history of chronic or recurrent pancreatitis, it is important to consider this hypothesis, especially if a known pseudocyst exists, in order to guide the diagnostic investigation and include an image exam that would allow its detection for its effective and in time treatment. Consequently, 
patients with known pseudocysts should have follow-up imaging in order to early detect of VPA thus allowing their elective treatment and decrease potencial fatal complications.

Classically, this entity was treated by open or laparoscopic surgery, involving ligation of the artery involved with or without reconstruction and sometimes with resection of structures being associated with greater mobility and mortality. With the constant evolution of endovascular procedures, they have become the first option for the treatment of visceral pseudoaneurysms with high success rates and lower morbidity and mortality rates than conventional surgery.

\section{Conclusion}

In addition to open surgical repair and laparoscopic surgery, there are also endovascular procedures for the treatment of visceral pseudoaneurysms, so the vascular surgeon must be aware with the available strategies, taking into account the patient, the characteristics and location of the visceral pseudoaneurysm.

\section{Acknowledgments}

None.

\section{References}

1. Sumpio Bauer. Overview of visceral artery aneurysm and pseudoaneurysm. 2020

2. Sousa Joel, Costa Diogo, Mansilha Armando. Visceral artery aneurysms: review on indications and current treatment strategies. International angiology: a journal of the International Union of Angiology. 2019;38(5):381-394

3. Chaer Rabih A, Christopher J Abularrage, Dawn M Coleman, et al. The Society for Vascular Surgery clinical practice guidelines on the management of visceral aneurysms. Journal of vascular surgery. 2020;72(1):3S-39S.
4. Kwong Jonathan M, Rockman Caron B, Kashyap Vikram S. Splanchnic Artery Aneurysms. In Sidawy AN, et al., editors. Rutherford's Vascular Surgery and Endovascular Therapy. Philadelphia, United States: Elvesier. 2018. p. 1109-1113.

5. Tessier Deron J, William M Stone, Richard J Fowl, et al. Clinical features and management of splenic artery pseudoaneurysm: case series and cumulative review of literature. Journal of vascular surgery. 2003;38(5):969-974.

6. Volpi Mauricio A, Eduardo Voliovici, Fernando Pinato, et al Pseudoaneurysm of the gastroduodenal artery secondary to chronic pancreatitis. Annals of vascular surgery. 2010;24(8):1136.e7-1136.e11.

7. Gilles J Hoilat, George Mathew, Hira Ahmad. Pancreatic Pseudoaneurysm. Treasure Island, FL: StatPearls; 2019.

8. Lu Minghao, Clifford Weiss, Elliot K Fishman, et al. Review of visceral aneurysms and pseudoaneurysms. Journal of computer assisted tomography, 2015;39(1):1-6.

9. Sumpio, Bauer. Treatment of visceral artery aneurysm and pseudoaneurysm. 2020.

10. Obara Hideaki, Matsubara Kentaro, Masanori Inoue, et al. Current management strategies for visceral artery aneurysms: an overview. Surgery today. 2020;50(1):38-49.

11. Tulsyan, Nirman, Vikram S Kashyap, Roy K Greenberg, et al. The endovascular management of visceral artery aneurysms and pseudoaneurysms. Journal of vascular surgery. 2007;45(2):276-283.

12. Rivera Philip A, Dattilo Jeffery B. Pseudoaneurysm. 2019. 